\title{
Clinical Significance of Radical Surgery in the Treatment of Silent Corticotroph Adenoma
}

\author{
Junhyung Kim, M.D., ${ }^{1}$ Seon Jin Yoon, M.D., ${ }^{1}$ Ju Hyung Moon, M.D., ${ }^{1-3}$ Cheol Ryong Ku, M.D., Ph.D., ${ }^{2-4}$ \\ Se Hoon Kim, M.D., Ph.D., ${ }^{3,5}$ Eun Jig Lee, M.D., Ph.D., ${ }^{2-4}$ Sun Ho Kim, M.D., Ph.D., ${ }^{1-3}$ Eui Hyun Kim, M.D., Ph.D. ${ }^{1-3}$ \\ Department of Neurosurgery, Yonsei University College of Medicine, Seoul, Korea \\ Endocrine Research Institute, ${ }^{2}$ Yonsei University College of Medicine, Seoul, Korea \\ Pituitary Tumor Center, ${ }^{3}$ Severance Hospital, Seoul, Korea \\ Department of Endocrinology, ${ }_{4}^{4}$ Yonsei University College of Medicine, Seoul, Korea \\ Department of Pathology, ${ }^{5}$ Yonsei University College of Medicine, Seoul, Korea
}

Objective : Silent corticotroph adenomas (SCA) are endocrine-inactive pituitary adenomas with positive immunohistochemistry staining for adrenocorticotropic hormone (ACTH). We investigated whether SCA-associated clinical profiles were more aggressive than hormonally negative adenomas (HNA).

Methods : Among 627 patients with pathologically proven endocrine-inactive pituitary adenomas between 2004 and 2013, positive immunohistochemistry revealed 55 SCAs and 411 HNAs. Surgical outcomes and radiological and endocrinological characteristics were compared.

Results : Strong female predominance was observed in the SCA group $(p<0.001)$. Cavernous sinus invasion was identified in 22 (40\%) SCA patients and $72(17.6 \%)$ HNA patients $(p<0.001)$. There were no differences in ACTH or cortisol levels between the two groups. The incidence of preoperative hypopituitarism and postoperative hormonal outcome did not differ between two groups. Total resection was achieved in 35 patients (63.7\%) with SCA and 332 patients $(80.8 \%)$ with HNA $(p=0.007)$. When tumors were completely removed, recurrence rates were not statistically different between two groups $(p=0.60)$. When complete resection was not achieved, tumors regrew from these remnants in seven patients (35.0\%) with SCA and 12 patients (15.2\%) with HNA ( $p=0.05)$.

Conclusion : Total surgical resection for SCA is often challenging as these tumors frequently invade a cavernous sinus. Early remnant tumor intervention is justified, because untreated residual pituitary tumors regrow when patients were followed up for a long time. Prophylactic radiotherapy is not warranted for completely resected SCAs as tumor recurrence is uncommon.

Key Words : Adrenocorticotropic hormone · Corticotrophs · Pituitary neoplasms.

\section{INTRODUCTION}

Pituitary adenomas are the third most common brain tu- mor, requiring different treatment strategies based on their hormonal activities. When a tumor does not produce any hormones, patients remain asymptomatic until the tumor be-

\footnotetext{
- Received : January 31, 2018 •Revised : April 23, 2018 •Accepted : April 29, 2018

- Address for reprints : Eui Hyun Kim, M.D., Ph.D.

Department of Neurosurgery, Yonsei University College of Medicine, 50-1 Yonsei-ro, Seodaemun-gu, Seoul 03722, Korea

Tel : +82-2-2228-2165, Fax : +82-2-393-9979, E-mail : euihyunkim@yuhs.ac
}

This is an Open Access article distributed under the terms of the Creative Commons Attribution Non-Commercial License (http://creativecommons.org/licenses/by-nc/4.0) which permits unrestricted non-commercial use, distribution, and reproduction in any medium, provided the original work is properly cited. 
comes a macroadenoma, presenting a mass effect over an optic apparatus, the hypothalamus, and adjacent blood vessels. Although clinically non-functioning pituitary adenomas (NFPAs) comprise about $25 \%$ of all pituitary adenomas, they are quite heterogeneous in nature, as they include null cell adenomas, various types of silent adenomas and even gonadotroph adenomas.

Silent corticotroph adenomas (SCAs) are a subtype of NFPAs which exhibit positive immunoreactivity for adrenocorticotropic hormone $(\mathrm{ACTH})$ without the clinical or biochemical features of hypercortisolism. Hormonally negative adenomas (HNAs) refer to null cell adenomas, which are negative for any immunohistochemical staining. SCAs comprise $2.9-5.7 \%$ of pituitary adenomas in surgical series ${ }^{2,5)}$. Previous reports suggest that the high rate of SCA recurrence and regrowth from residual tumor remnants may necessitate adjuvant treatments, such as radiation therapy ${ }^{13,15)}$.

Based on our experience with 55 SCA cases, we clinically characterized SCAs and evaluated the surgical outcome of SCAs compared with HNAs.

\section{MATERIALS AND METHODS}

\section{Patients}

A total of 627 patients with NFPAs treated surgically at our institution from 2004 until 2013, were recruited from our pituitary tumor database and analyzed retrospectively. Reports on immunohistochemical staining were available for 620 patients. Gonadotroph adenomas and other silent adenomas were excluded from this study based on immunohistochemical staining for pituitary hormones. Fifty-five SCA cases and 411 HNA cases were included and compared. The diagnoses of SCAs were based on the lack of clinical symptoms of hypercortisolism, the lack of laboratory data consistent with hypercortisolism, and the finding of positive ACTH immunoreactivity. This paper was reviewed and approved by Severance Hospital Institutional Review Board.

\section{Radiological evaluation}

All patients underwent preoperative dynamic magnetic resonance imaging (MRI) of the sellar region, using a 1.5-Tesla system (Signa; General Electric, Minneapolis, MN, USA) or a 3.0-Tesla system (Achieva; Philips, Best, The Netherlands).
The MRI evaluation included spin-echo T1-weighted (TR 500 ms/TE 20 ms) imaging, T2-weighted (TR 3500 ms/TE 100 ms) imaging, and dynamic contrast-enhanced (TR 400 ms/TE 10 ms) imaging after intravenous administration of gadoliniumdiethylenetriamine pentaacetic acid $(0.1 \mathrm{~mL} / \mathrm{kg}$ of body weight) with a temporal resolution of 25 seconds. Immediate postoperative 3-Tesla diagnostic MRI was performed within 48 hours after surgery; the radicality of tumor removal was determined. Follow-up MRI was performed 1 year after surgery and every other year thereafter.

All adenomas were classified according to a modified Hardy radiological classification scheme ${ }^{8,9)}$. Type I was confined entirely within the sellae and had a size $<1 \mathrm{~cm}$. Type II extended into the suprasellar space, up to $<1 \mathrm{~cm}$ above the line of the diaphragm. Type III extended into the suprasellar area, up to $>1 \mathrm{~cm}$ above the line of the diaphragm, or extended into the sphenoid sinus. Type IV was defined as a tumor that invaded the cavernous sinus. In addition, Hardy type IV lesions were further classified according to the Knosp classification to demonstrate the degree of cavernous sinus invasions.

Extent of resection, recurrence of the tumor, and regrowth of the remnant were determined based on postoperative MRI findings, which were judged by a neurosurgeon and a radiologist. The size of the tumor was defined as the longest diameter of the tumor, as seen in the coronal MRI view.

\section{Endocrinological evaluation}

Basal serum levels of pituitary hormones (including prolactin, luteinizing hormone [LH], follicle-stimulating hormone [FSH], thyroid-stimulating hormone [TSH], growth hormone [GH], and ACTH) along with cortisol, T3, free T4, insulin-like growth factor-1, and estradiol or testosterone were checked at 8:00 am before surgery and 1 week, and 1, 3, 6, and 12 months after surgery. Preoperative serum cortisol levels and 24-hour urinary free cortisol levels were compared between patients having SCA and those with HNA to evaluate the baseline levels and subgroup differences. Preoperative serum cortisol and ACTH levels were compared to postoperative hormone levels in the SCA group to determine if surgery affected hormonal regulation. The HNA group was analyzed in the same manner to determine if there was a procedural effect on hormonal regulation.

Endocrinological assessment with a combined pituitary function test (CPFT) was fully described in our previous report ${ }^{10)}$. TSH levels were measured by chemiluminescent microparticle 
immunoassay using ARCHITECT TSH Reagent kit (Abbott Laboratories, Longford, Ireland). LH, FSH, prolactin, and cortisol levels were all measured by sandwich ELISA using Access Reagent Pack (Backman Coulter Inc., Brea, CA, USA). GH levels were measured by chemiluminescence immunoassay using LIAISON hGH Reagent (DiaSorin, Saluggia, Italy), and ACTH levels were measured by electrochemiluminescence immunoassay (Roche Diagnostics, Mannheim, Germany). The results for both preoperative and postoperative CPFT were available for 303 patients (34 patients with SCA and 269 patients with HNA). The CPFT was interpreted as described below and the results were confirmed by two endocrinologists. After intravenous injection of $0.1 \mathrm{U} / \mathrm{kg}$ regular insulin, thyrotropin-releasing hormone $(500 \mathrm{mcg})$, and LH-releasing hormone $(100 \mathrm{mcg})$, patients were evaluated serially for 2 hours for their responses of serum GH, cortisol, ACTH, LH, FSH, $\mathrm{TSH}$, and prolactin (PRL). The criteria for normal responses were as follows : peak GH level increased by $>3 \mathrm{ng} / \mathrm{mL}$, peak cortisol increased by $>180 \mathrm{ng} / \mathrm{mL}$ or $>70 \mathrm{ng} / \mathrm{mL}$ compared with basal level, basal PRL level was 2-15 ng/mL, and peak PRL level increased $>2$-fold of basal PRL level, peak TSH level increased greater than $5 \mathrm{IU} / \mathrm{mL}$ compared with basal level and free T4 was in the normal range, peak FSH increased $>2 \mathrm{mIU} / \mathrm{mL}$ compared with basal level, and peak LH increased greater than $10 \mathrm{mIU} / \mathrm{mL}$ compared with basal level. Elevated levels of basal gonadotropins in postmenopausal patients and no menstrual disorders in premenopausal patients were regarded as normal. Changes in pituitary function before and after transsphenoidal approach (TSA) were classified into four categories : 1) normal to normal (no pituitary hormone deficiency before and after TSA); 2) improved hypopituitarism (hypopituitarism improved, including complete recovery); 3) persistent hypopituitarism (the degree of pituitary hormone deficiency did not change after TSA); and 4) worsened hypopituitarism (the number and/or degree of pituitary hormone deficiency worsened after TSA). The last of these categories included the new development of postoperative hypopituitarism and the aggravation of preoperatively existing hypopituitarism. CPFT was performed to evaluate anterior pituitary function before surgery, 6-12 months after surgery, and every 2 years thereafter.

\section{Surgical procedure}

The surgical procedure was performed in the conventional microscopic TSA by two neurosurgeons (S.H.K. and E.H.K.). After dural opening, we first localized the normal pituitary gland and checked for tumor consistency and the presence of a pseudocapsule. During tumor removal, we made every effort to preserve a normal remaining pituitary gland whereas the pseudocapsule was always removed as radically as possible. With any faint suspicion of dural invasion, all exposed dura mater was removed as much as possible. In five cases with giant pituitary macroadenomas, the transcranial approach was performed for better tumor visualization and removal as the initial treatment. For the tumors invading a cavernous sinus, the tumor was removed as much as possible through a window on the medial wall of the cavernous sinus. However, for endocrine-inactive tumors, the direct approach to the lateral compartment of a cavernous sinus was not performed. When cerebrospinal fluid (CSF) leakage was noted after tumor removal, it was sealed by various direct closure techniques to close the leaking point. Postoperative lumbar drainage of CSF was performed in many of our early cases; however, it has not been used since 2005. Most of the remnant tumors were only observed until regrowth was evident on serial follow-up MRIs. Secondary treatment options included re-operation, gamma knife radiosurgery and radiotherapy.

\section{Statistical analysis}

For categorical analysis, a chi square test and Fisher's exact test were used. Survival analysis was performed by KaplanMeier method in order to demonstrate progression-free survival after resection for each subgroup and to compare the outcome related to the suspected risk factors. All analyses were conducted using IBM SPSS Statistics (version 20.0; IBM, Armonk, NY, USA). $p$ values less than 0.05 were considered to be statistically significant.

\section{RESULTS}

\section{Clinical characteristics}

The clinical characteristics of our 466 patients are summarized in Table 1. The median follow-up duration was not statistically different between the SCA and HNA groups (SCA, 20.0 months; HNA, 31.0 months). The mean age at diagnosis was 49.7 years in 55 patients with SCA and 49.8 years in 411 patients with HNA, which was not statistically different $(p=0.96)$. The SCA group included 11 males and 44 females, whereas the HNA group included 196 males and 215 females. 
The male-to-female ratio $(11: 44)$ in the SCA group revealed female dominance in this subtype compared with the HNA group $(p<0.001)$.

The mean tumor size during preoperative MRI was $28.6 \pm 11.8$ $\mathrm{mm}$ and $27.0 \pm 8.9 \mathrm{~mm}$ for SCAs and HNAs, respectively; there was no intergroup difference $(p=0.46)$. To evaluate cavernous sinus invasion of the tumor, we primarily depended on preoperative MRI images, but surgical findings played decisive roles when cavernous sinus invasions were noted during the surgeries even if the preoperative MRI could not confirm the invasion. In the SCA group, cavernous sinus invasion was detected on preoperative MRI in 22 patients (40\%) among whom one patient was Knosp grade II, four were Knosp grade III, and 17 were Knosp grade IV, whereas only 72 patients (17.5\%) with HNA displayed cavernous invasion, among whom one patient was Knosp grade I, 10 were Knosp grade II, 32 were Knosp grade III and 29 were Knosp grade IV. SCA group revealed a higher rate of a cavernous sinus invasion $(p<0.001)$.

\section{Endocrinological outcome}

Preoperative and postoperative hormone levels in the SCA and HNA groups are summarized in Table 2. No statistically significant differences were found between the SCA and HNA groups, either preoperatively or postoperatively. Serum ACTH and cortisol levels decreased in both groups whereas the 24hour urinary free cortisol levels rather increased postoperatively.

Preoperatively, the incidence of hypopituitarism was not different identified between two groups (64.7\% in SCA group vs. $74.3 \%$ in HNA group, $p=0.16$ ). Comparisons between preoperative and postoperative CPFT were available in 303 patients (Table 3). Overall, normal pituitary function was preserved in 69 (22.8\%), improved in 146 (48.2\%), persistent in 48 (15.8\%), and aggravated in 40 patients (13.2\%). No statistically significant differences were found between the SCA and HNA groups $(p=0.584)$.

Table 1. Patient demographics

\begin{tabular}{lccc}
\hline & SCA & HNA & p-value \\
\hline Number of cases & 55 & 411 & $<0.001^{*}$ \\
Gender & 11 & 196 & 215 \\
$\quad$ Male & 44 & 49.8 & 0.96 \\
Female & 49.7 & $31.0(3-122)$ & 0.881 \\
Mean age (years) & $20.0(3-122)$ & $27.0 \pm 8.9$ & 0.46 \\
Duration of follow up (months) & $28.6 \pm 11.8$ & $72(17.5)$ & $<0.001^{*}$ \\
Size of tumor (mm) & $22(40)$ & \\
Cavernous sinus invasion & & \\
\hline
\end{tabular}

Values are presented as mean \pm standard deviation, number (\%), or number (range). * Statistically significant. SCA : silent corticotroph adenoma, HNA : hormonally negative adenoma

Table 2. Preoperative and postoperative hormone level

\begin{tabular}{|c|c|c|c|}
\hline & SCA & HNA & $p$-value \\
\hline \multicolumn{4}{|l|}{ АСТH (pg/mL) } \\
\hline Preoperative & 48.8 & 36.4 & 0.09 \\
\hline Postoperative & 24.2 & 23.1 & 0.75 \\
\hline \multicolumn{4}{|c|}{ Serum cortisol (mcg/dL) } \\
\hline Preoperative & 12.6 & 11.4 & 0.18 \\
\hline Postoperative & 9.9 & 10.5 & 0.51 \\
\hline \multicolumn{4}{|c|}{ 24-hours urinary free cortisol (mcg/dL) } \\
\hline Preoperative & 184.2 & 183.3 & 0.98 \\
\hline Postoperative & 292.5 & 292.6 & 0.99 \\
\hline
\end{tabular}

SCA : silent corticotroph adenoma, HNA : hormonally negative adenoma 


\section{Surgical outcome}

Surgical outcome is summarized in Table 4. The transcranial approach was performed as initial treatment in two SCA cases and three HNA cases. Total resection was achieved in $63.7 \%$ of SCA patients and in $80.8 \%$ of HNA patients $(p=0.007)$. When cavernous sinus was invaded, total resection was possible in $27.3 \%$ of SCA patients and in $23.6 \%$ of HNA patients. Postoperative CSF rhinorrhea developed in two SCA cases and four HNA cases $(p=0.15)$.

Results regarding tumor recurrence and tumor remnant regrowth are shown in Table 5. In the HNA group, follow-up MRIs detected recurrent tumors in five patients (1.2\%) whose tumors were previously completely resected; no patients with SCA experienced tumor recurrence $(p=0.41)$. Serial follow-up
MRIs demonstrated regrowth in seven of 20 patients $(35.0 \%)$ with remnant SCA and in 12 of 79 patients (15.2\%) with remnant HNA $(p=0.05)$. Time to progression from the initial operation between the two subgroups did not show statistically significant difference, with 49 months in the SCA group and 46 months in the HNA group $(p=0.56)$. All patients with regrown remnant tumors required adjuvant treatments, including re-operation, gamma knife surgery, and conventional radiotherapy. Actuarial progression-free survival at 12, 24, and 48 months after operation was $97.4 \%, 90.2 \%$, and $84.9 \%$ in the SCA group, and $99.1 \%, 98.5 \%$, and $96.2 \%$ in the HNA group, respectively ( $p=0.009$ ) (Fig. 1). Although SCAs showed more invasive feature with more frequent recurrence, recurrence was not common once total removal as achieved (Fig.

Table 3. Postoperative hormonal outcome

\begin{tabular}{lccc}
\hline Postoperative hormonal outcome & SCA & HNA & $p$-value \\
\hline Preserved normal pituitary function $(\mathrm{n}=69,22.8 \%)$ & $9(26.5)$ & $60(22.3)$ & 0.66 \\
Improved hypopituitarism $(\mathrm{n}=146,48.2 \%)$ & $14(41.2)$ & $132(49.1)$ & 0.47 \\
Persistent hypopituitarism $(\mathrm{n}=48,15.8 \%)$ & $6(17.6)$ & $42(15.6)$ & 0.80 \\
Aggravation of hypopituitarism $(\mathrm{n}=40,13.2 \%)$ & $5(14.7)$ & $35(13.0)$ & 0.78 \\
Total $(\mathrm{n}=303)^{*}$ & 34 & 269 & 0.854 \\
\hline
\end{tabular}

Values are presented as number (\%). *Total number of patients in whom comparison between preoperative and postoperative combined pituitary function test was available. SCA : silent corticotroph adenoma, HNA : hormonally negative adenoma

Table 4. Surgical outcome

\begin{tabular}{lccc}
\hline & SCA & HNA & p-value \\
\hline Surgical approach & & & 0.11 \\
Transsphenoidal approach & $53(96.4)$ & $408(99.2)$ & $3(0.7)$ \\
Transcranial approach & $2(3.6)$ & $332(80.8)$ & $0.007^{*}$ \\
Total resection & $35(63.7)$ & $4(1)$ & 0.15 \\
Postoperative CSF rhinorrhea & $2(4)$ & \\
\hline
\end{tabular}

Values are presented as number (\%). *Statistically significant. SCA : silent corticotroph adenoma, HNA : hormonally negative adenoma, CSF : cerebrospinal fluid

Table 5. Recurrence and regrowth

\begin{tabular}{lccc} 
& SCA & HNA & p-value \\
\hline Remnant growing* & $7(35.0)$ & $12(15.2)$ & 0.05 \\
Recurrence (cases) & 0 & $5(1.2)$ & 0.41 \\
Time to progress (months) & $49(11-89)$ & $46(11-100)$ & 0.56 \\
Second treatment & Reoperation 4 & Reoperation 5 & GKS 9 \\
& GKS 2 & Radiotherapy 1 & Radiotherapy 1 \\
\hline
\end{tabular}

Values are presented as number (\%) or number (range) unless otherwise indicated. *This indicates the number of patients with adenoma regrown from residual tumor after subtotal resection. SCA : silent corticotroph adenoma, HNA : hormonally negative adenoma 


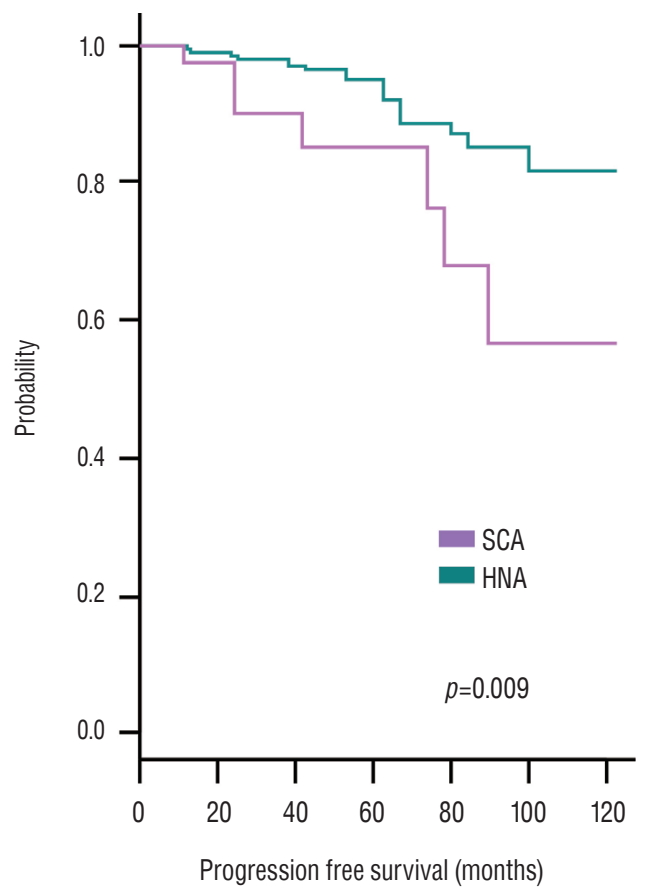

Fig. 1. Progression-free survival of nonfunctioning pituitary adenomas. The SCA group has a reduced survival outcome when compared with the HNA group $(p=0.009)$. The SCA group's actuarial survival rate at 12,24 , and 48 postoperative months was $97.4 \%, 90.2 \%$, and $84.9 \%$, respectively. HNA : hormonally negative adenoma, SCA : silent corticotroph adenoma.
2A). In 55 patients with SCA, the progression-free survival curve showed a high probability of regrowth when total resection was not achieved (Fig. 2B).

\section{Case illustration}

A 51-year old male patient presented with a symptom of visual disturbance. He was a hepatitis B-virus carrier without any other significant medical history. Visual field evaluation revealed that the patient has a typical bitemporal hemianopsia. Brain MRI showed a pituitary macroadenoma with significant optic nerve compression and right cavernous sinus invasion (Fig. 3A). Tumor was removal with TSA leaving a small remnant in right cavernous sinus (Fig. 3B). Visual field defect improved after surgical treatment. A year after the surgery, MRI revealed increase in the size of residual tumor in the right cavernous sinus (Fig. 3C), which was then irradiated by Gamma Knife radiosurgery (GKS) with 14 Gy of margin dose. The tumor was under control until 7-year post-GKS MRI revealed recurrent tumor in the intrasellar and suprasellar space as well as inside right cavernous sinus (Fig. 3D). The recurrent tumor was removed by two times of surgery; extended TSA for intrasellar and right cavernous sinus exploration and su-
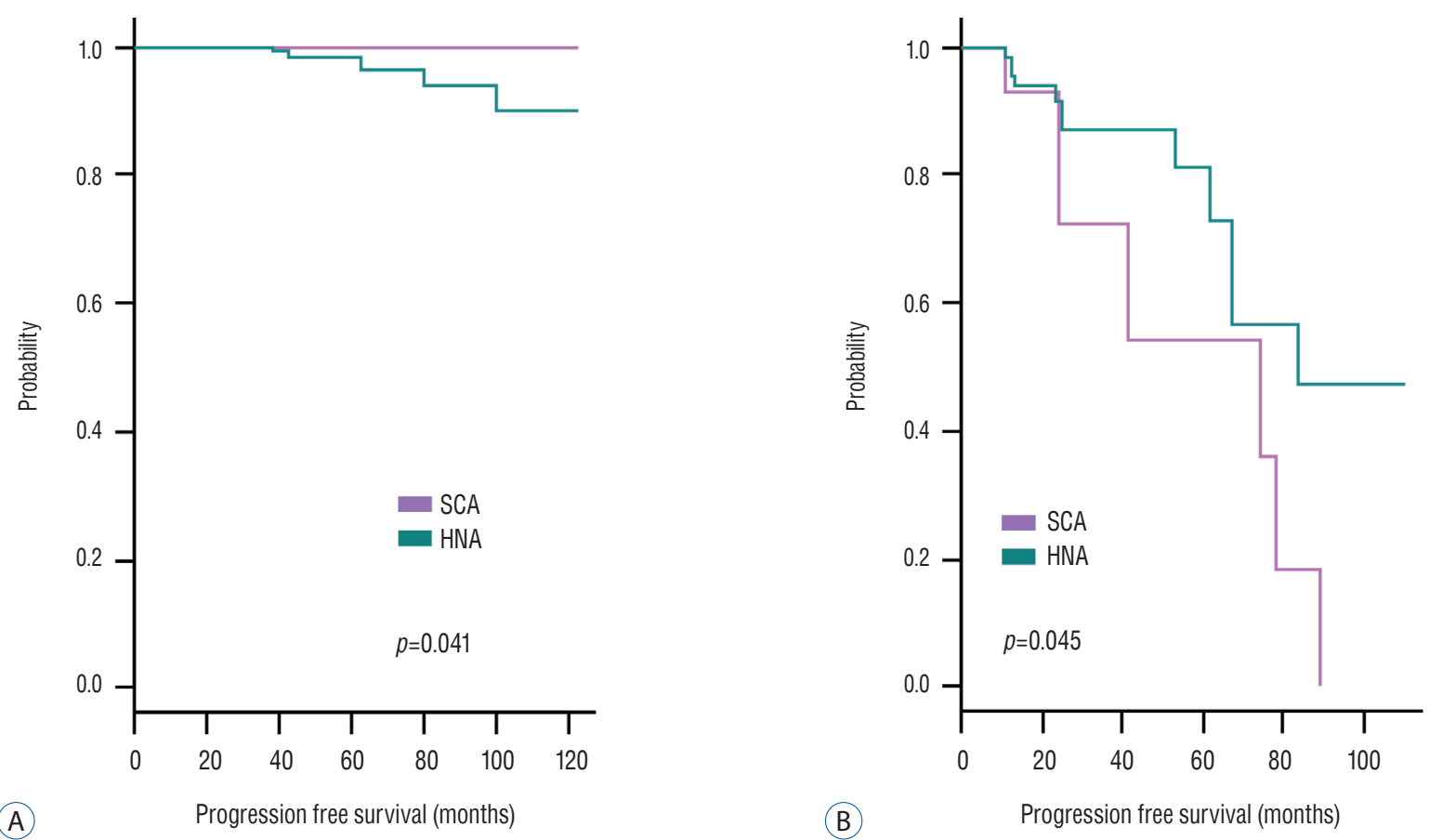

Fig. 2. Kaplan-Meyer curve of progression-free survival in 55 patients with SCA. A : The extent of resection was a strong prognostic factor) as no patient experienced recurrence when their tumors were completely removed. $\mathrm{B}$ : When tumors were not completely removed, SCAs showed worse prognosis compared with HNAs. HNA : hormonally negative adenoma, SCA : silent corticotroph adenoma. 

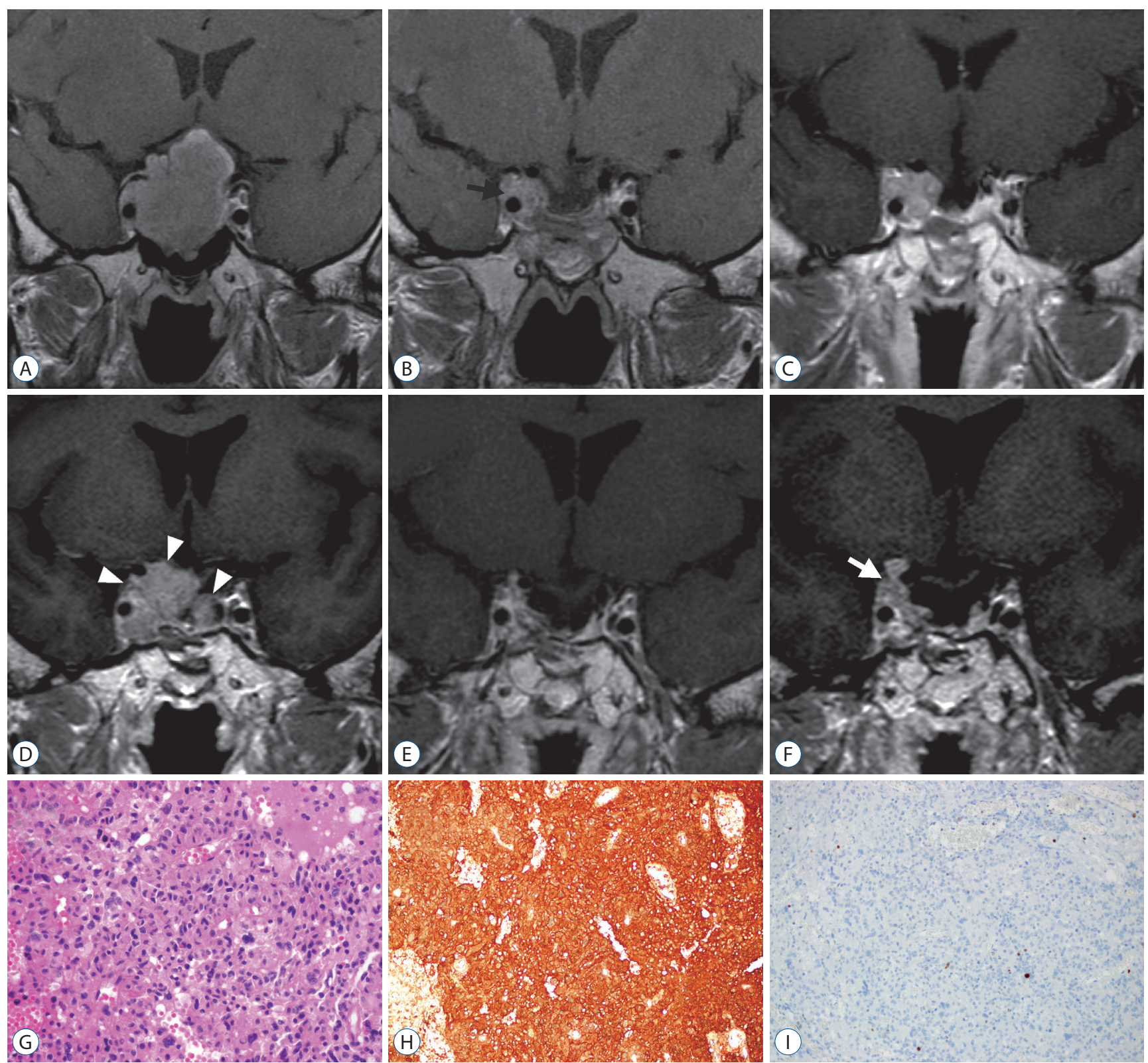

Fig. 3. Illustrative case with a silent corticotroph adenoma. A 51-year old male patient was diagnosed as a pituitary macroadenoma with significant optic nerve compression and right cavernous sinus invasion (A). Tumor was removal with transsphenoidal approach leaving a small remnant in right cavernous sinus (black arrow, B). A year after the surgery, MRI revealed increase in the size of residual tumor in the right cavernous sinus (C). After GKS, the tumor was under control until 7-year post-GKS MRI revealed recurrent tumor in the intrasellar and suprasellar space as well as inside right cavernous sinus (white arrowheads, D). After radical removal of the recurrent tumor, MRI revealed no visible tumor (E), however, 3 years later, recurrent tumor was found again inside right cavernous sinus and suprasellar space (white arrow, F). Histopathological examination demonstrated a pituitary adenoma (G; $\mathrm{H} \& \mathrm{E}, \times 400)$ with positive immunohistochemistry for adrenocorticotropic hormone $(\mathrm{H} ; \mathrm{ACTH}, \times 200)$. Ki-67 labeling index was only $1-2 \%(\mathrm{I} ; \mathrm{Ki}-67, \times 200)$. MRI : magnetic resonance imaging, GKS : Gamma Knife radiosurgery, ACTH : adrenocorticotropic hormone.

praorbital keyhole transcranial approach for the removal of suprasellar tumor. After radical removal of the recurrent tumor, MRI revealed no visible tumor (Fig. 3E). Histopathological findings were consistent with a pituitary adenoma on $\mathrm{H} \& \mathrm{E}$ stain and immunohistochemistry for ACTH was positive (Fig.
3G-I). Despite aggressive surgical resection, recurrent tumor was found inside right cavernous sinus and suprasellar space on the MRI taken 3 years after second surgery (Fig. 3F), which necessitated 2nd GKS. 


\section{DISCUSSION}

\section{Silent pituitary macroadenomas and their char- acteristics}

The female dominance in the SCA group is a very interesting finding in our series, which is also very distinct characteristic of Cushing's disease. This finding has not been previously reported in a major SCA series. When we consider that both SCA and Cushing's disease originate from corticotroph cells and share similar molecular pathogenesis involving proopiomelanocortin and prohormone convertase $1 / 3^{7)}$, female dominance in SCA is not unexpected.

There have been many reports suggesting that SCAs are more likely to invade adjacent anatomical structures, such as a cavernous sinus ${ }^{1,2,6,7,12,13)}$. In our study, the incidence of cavernous sinus was $40.0 \%$ in patients with SCA, which is much higher than the incidence seen in patients with HNA (17.5\%). However, there was no difference in patient age or tumor size.

\section{Endocrinological aspect}

ACTH and cortisol levels were slightly higher in the SCA group than in the HNA group; however, this difference was not statistically significant. We excluded the possibility that the SCA group included cases of ACTH-secreting pituitary adenoma (Cushing's disease) based on preoperative measurement of 24-hour urinary free cortisol. ACTH and cortisol levels did not differ after surgery. Both serum ACTH levels and serum cortisol hormone levels were somewhat decreased after surgery, which may be the result of surgical traumatization on the normal pituitary gland. Urine cortisol levels showed no statistically significant intergroup difference between each groups' preoperative and postoperative status. The 24-hour urinary free cortisol levels were rather increased after surgery in both SCA and HNA groups. As the level of 24-hour urinary free cortisol largely depends on BMI and co-morbidities such as diabetes, further well controlled in-depth study might be necessary to clarify this confusing result.

It has been reported that hypopituitarism more commonly develops in patients with $\mathrm{SCA}^{6}$. However, our result did not indicate a higher incidence of hypopituitarism in the SCA group, but rather a slightly higher incidence in the HNA group. After surgery, hormonal outcomes were judged by comparing preoperative and postoperative CPFT, which revealed no differences between the SCA and HNA groups.

\section{Recurrence and regrowth}

In our series, the chance of total resection was much lower in the SCA group than in the HNA group, which is consistent with the more frequent preoperative cavernous sinus invasion by SCAs. Although SCAs have a higher recurrence risk ${ }^{1,2,6,712,13)}$, the recurrence rate in our series was not significantly higher than that of other NFPAs when tumors were completely removed $(p=0.60)$. Several studies report higher recurrence rates after surgical resection ${ }^{13,14)}$ but their results lack a comparative group with other pituitary tumor resections. Bradley et al. ${ }^{3)}$ found that SCA recurrence rate was not higher compared with NFPA, but they did not distinguish between regrowth and recurrence. In this study, remnant regrowth was distinguished from true recurrence after total resection; the regrowth rate was $35 \%$, which does not differ greatly from other reports ${ }^{3,4)}$. The low recurrence rate may be related to our surgical strategy to remove all entities related to the pituitary tumor, including the tumor capsule ${ }^{9,10)}$. Especially, when pituitary pseudocapsule was think enough to be used as a surgically available dissection plane, en bloc tumor resection was always tried. And, even when tumors were remove in piecemeal fashion, pseudocapsule was removed as radical as possible.

It is very important to be aware that SCAs are not uncommon, as they comprise $8.8 \%$ (55 of 620 cases) of all endocrineinactive pituitary adenomas. Because detailed hormonal diagnosis based on immunohistochemistry is only available after surgery, radical tumor resection should be attempted whenever possible. Traditionally, surgical resection has frequently been followed by radiation in order to lower the recurrence rate. However, this study suggests that prophylactic radiation is not necessary when SCAs are completely removed. This is also supported by other studies, which suggest that the role of irradiation needs to be reassessed for its low recurrence rate, particularly when tumors are resected completely ${ }^{1,11)}$.

It is noteworthy that the SCA regrowth rate from remnant tumors was higher than that of HNA in this study. When remnants persisted after surgery, the overall regrowth rate was $35.0 \%$ for SCAs, while its 8-year progression-free survival was only 31\%. Therefore, rather than a "wait and see" approach, more meticulous follow up is mandatory and early intervention with radiosurgery or radiotherapy can be recommended. 


\section{CONCLUSION}

Total surgical resection of SCAs is often impossible as these tumors frequently invade a cavernous sinus. As untreated residual SCAs have a higher chance to regrow, more meticulous follow up is mandatory and early intervention on remnant tumors is recommended. Complete tumor removal is critical in the treatment of SCAs as it dramatically decrease the risk of recurrence, and thus, radical surgical resection should be attempted whenever possible, even for endocrine-inactive pituitary adenomas. Prophylactic radiotherapy is not warranted for SCAs that have been completely removed, as tumor recurrence is rare.

\section{CONFLICTS OF INTEREST}

No potential conflict of interest relevant to this article was reported.

\section{INFORMED CONSENT}

Informed consent was obtained from all individual participants included in this study.

\section{- Acknowledgements}

This study was supported by a faculty research grant of Yonsei University College of Medicine (6-2017-0040).

The authors thank Juyoon Park, R.N., M.P.H., O.C.N., Min Kyeong Jang, R.N., Sung Ja Kang, R.N., and Bok Soon Lee, R.N., Sujin Ryu, R.N., Pituitary Tumor Center and Soo Yeon Choi, M.P.H., Department of Medical Recording for their tremendous effort in performing the endocrinological tests and data acquisition for such a long follow-up duration.

\section{References}

1. Alahmadi H, Lee D, Wilson JR, Hayhurst C, Mete O, Gentili F, et al. : Clinical features of silent corticotroph adenomas. Acta Neurochir (Wien) 154 : 1493-1498, 2012
2. Baldeweg SE, Pollock JR, Powell M, Ahlquist J : A spectrum of behaviour in silent corticotroph pituitary adenomas. Br J Neurosurg 19 : 38-42, 2005

3. Bradley KJ, Wass JAH, Turner HE : Non-functioning pituitary adenomas with positive immunoreactivity for $\mathrm{ACTH}$ behave more aggressively than ACTH immunonegative tumours but do not recur more frequently. Clin Endocrinol (0xf) 58 : 59-64, 2003

4. Cho HY, Cho SW, Kim SW, Shin CS, Park KS, Kim SY : Silent corticotroph adenomas have unique recurrence characteristics compared with other nonfunctioning pituitary adenomas. Clin Endocrinol (Oxf) 72 : 648653, 2010

5. Horvath E, Kovacs K, Killinger DW, Smyth HS, Platts ME, Singer W : Silent corticotropic adenomas of the human pituitary gland: a histologic, immunocytologic, and ultrastructural study. Am J Pathol 98 : 617-638, 1980

6. Ioachimescu AG, Eiland L, Chhabra VS, Mastrogianakis GM, Schniederjan MJ, Brat D, et al. : Silent corticotroph adenomas: Emory University cohort and comparison with $\mathrm{ACTH}$-negative nonfunctioning pituitary adenomas. Neurosurgery 71 : 296-303; discussion 304, 2012

7. Jahangiri A, Wagner JR, Pekmezci M, Hiniker A, Chang EF, Kunwar $S$, et al. : A comprehensive long-term retrospective analysis of silent corticotrophic adenomas vs hormone-negative adenomas. Neurosurgery 73 : 8-17; discussion 17-18, 2013

8. Kim EH, Ku CR, Lee EJ, Kim SH : Extracapsular en bloc resection in pituitary adenoma surgery. Pituitary $18:$ 397-404, 2015

9. Ku CR, Kim EH, Oh MC, Lee EJ, Kim SH : Surgical and endocrinological outcomes in the treatment of growth hormone-secreting pituitary adenomas according to the shift of surgical paradigm. Neurosurgery 71(2 Suppl Operative) : ons192-ons203; discussion ons203, 2012

10. Lee EJ, Ahn JY, Noh T, Kim SH, Kim TS, Kim SH : Tumor tissue identification in the pseudocapsule of pituitary adenoma: should the pseudocapsule be removed for total resection of pituitary adenoma? Neurosurgery 64 : ons62-ons69; discussion ons69-ons70, 2009

11. Lillehei KO, Kirschman DL, Kleinschmidt-DeMasters BK, Ridgway EC : Reassessment of the Role of Radiation Therapy in the Treatment of Endocrine-inactive Pituitary Macroadenomas. Neurosurgery 43 : 432 438; discussion 438-439, 1998

12. Pawlikowski M, Kunert-Radek J, Radek M : "Silent"corticotropinoma. Neuro Endocrinol Lett 29 : 347-350, 2008

13. Scheithauer BW, Jaap AJ, Horvath E, Kovacs K, Lloyd RV, Meyer FB, et al. : Clinically silent corticotroph tumors of the pituitary gland. Neurosurgery 47 : 723-729; discussion 729-730, 2000

14. Webb KM, Laurent JJ, Okonkwo DO, Lopes MB, Vance ML, Laws ER Jr : Clinical characteristics of silent corticotrophic adenomas and creation of an internet-accessible database to facilitate their multi-institutional study. Neurosurgery 53 : 1076-1084; discussion 1084-1075, 2003

15. Xu Z, Ellis S, Lee CC, Starke RM, Schlesinger D, Lee Vance M, et al. : Silent corticotroph adenomas after stereotactic radiosurgery: a casecontrol study. Int J Radiat Oncol Biol Phys 90 : 903-910, 2014 